\title{
On a Class of Polynomials Connected with the Korteweg-deVries Equation*
}

\author{
M. Adler ${ }^{1}$ and J. Moser ${ }^{2}$ \\ 1 University of Wisconsin, Madison, Wisconsin 53706, USA \\ 2 New York University, New York, New York 10012, USA
}

\begin{abstract}
A new and simpler construction of the family of rational solutions of the Korteweg-deVries equation is given. This construction is related to a factorization of the Sturm-Liouville operators into first order operators and a new deformation problem for the latter. In the final section the spectral representation for the corresponding complex potentials is discussed.
\end{abstract}

\section{1.}

In [1] special classes of solutions of the Korteweg-deVries equation

$$
u_{t}=3 u u_{x}-\frac{1}{2} u_{x x x}=X_{2} u
$$

were studied, in particular all those $u=u(x, t)$ which are rational functions of $x$ for each value of $t$. It turns out that these solutions are rational functions of $t$ as well and of very special structure. In this paper we give a new construction of these solutions with emphasis on their algebraic properties.

To describe the family of rational solutions of (1.1), one does well to introduce the sequence of associated Korteweg-deVries equations

$$
u_{t}=X_{k}(u), \quad k=1,2, \ldots,
$$

which are related by

$$
X_{k}=\frac{\partial}{\partial x} \frac{\delta H_{k}}{\partial u}
$$

to the sequence of conserved quantities

$$
H_{k}=\int P_{k}\left(u, u^{\prime}, \ldots\right) d x
$$

associated with (1.1). These $X_{k}$ can be recursively defined by

$$
X_{k+1}(u)=\left(u \frac{\partial}{\partial x}+\frac{\partial}{\partial x} u-\frac{1}{2}\left(\frac{\partial}{\partial x}\right)^{3}\right) \frac{\delta H_{k}}{\partial u}
$$

* This work was partially sponsored by the National Science Foundation, Grants MCS 77-01986 DMM3, MCS 75-17385, and by the United States Army under Contract No. DAAG29-75-C-0024 
as was shown originally by Lenard, see [3]. For the notation used here we refer to $[6,1]$.

The above nonlinear differentialoperators commute, and so do the flows $e\left(t_{k} X_{k}\right)$ generated by them. We ask for the manifold $M$ of rational functions vanishing at $x$ $=\infty$ invariant under all the flows $e\left(t_{k} X_{k}\right)$. It is one of the results of [1] that $M$ decomposes into denumerably many manifolds $M_{d}$ of dimensions $d$ for $d=1,2, \ldots$ and, moreover, each $M_{d}$ constitutes an orbit of the single function

$$
u=\frac{d(d+1)}{x^{2}}
$$

under the flows $e\left(\sum t_{k} X_{k}\right)$ (see [1], Section 3, Theorem 2).

In this paper we want to give a representation of $M_{d}$ in terms of a class of polynomials $\theta_{d}=\theta_{d}\left(\tau_{1}, \tau_{2}, \ldots, \tau_{d}\right)$ depending on $d$ variables. These polynomials will be defined in Section 2 and they allow the representation of all $u \in M_{d}$ in the form

$$
u_{d}(x)=-2\left(\frac{\partial}{\partial x}\right)^{2} \log \theta_{d}\left(\tau_{1}+x, \tau_{2}, \ldots, \tau_{d}\right)
$$

and this representation is one to one, so that $\tau_{1}, \tau_{2}, \ldots, \tau_{d}$ can be viewed as global coordinates on $M_{d}$. The $X_{k}$ give rise to vector fields $\Gamma_{k}$ on $M_{d}$ which are expressible in terms of the $\tau_{j}$, and in Section 5 we will determine these $\Gamma_{k}$. It turns out that the $\tau_{2}, \ldots, \tau_{d}$ can be subjected to a group of birational transformations

$$
\tau_{j}^{*}=a_{j} \tau_{j}+g_{j}\left(\tau_{1}, \ldots, \tau_{j-1}\right), \quad a_{j} \neq 0,
$$

$g_{j}$ being polynomials, without changing the above representation. Moreover, the parameters $\tau_{j}^{*}$ can be introduced so that

$$
\Gamma_{k}=\frac{\partial}{\partial \tau_{k}^{*}}
$$

i.e. that

$$
\frac{\partial u_{d}}{\partial \tau_{k}^{*}}=X_{k}(u) .
$$

In other words the $\tau_{k}^{*}$ can be identified with the $t$-variable $t_{k}$ of $X_{k}$. This picture was developed already in [1] but here this representation is made more explicit through (1.3) and the construction of the polynomials $\theta_{k}$. The representation (1.3) is an analogue to that of Its and Matveev [4] for the case of solutions of (1.1) having a fixed period in $x$ and for which the corresponding Hill's equation has only finitely many simple eigenvalues. It is conceivable that (1.3) could be obtained by a limit process from the formula [4], but we did not succeed in this way ${ }^{1}$. Similarly one may expect that (1.3) could be obtained as the limit of the $N$-soliton potential (see [8]). This is indeed the case as was shown by Ablowitz and Satsuma [17], who also considered other differential equations. The underlying idea to study rational solutions of partial differential equations and the corresponding motion of the poles was pursued by Calogero and the brothers Choodnovsky for a large variety of equations (see $[18,20]$ ).

1 H. P. McKean, via personal communication, informed us of his success in carrying out this approach 
We mention that the solutions of the type (1.3) for the case $d=2$ were considered by Moses $[9,16]$.

The construction of the $\theta_{k}$ as well as the proof of the above statements are based on a transformation of differential operators $L=-D^{2}+u$ into each other which is certainly not new (see $[2,11,12,21]$ ) and the so-called Miura transformation [7] for which we give a natural derivation. This derivation is based on the factorization

$$
L=A^{*} A ; \quad A=D-v,
$$

where $u=v^{\prime}+v^{2}$. This factorization will play a crucial role in the following derivation and we will attempt to tie it to isospectral deformations. Similarly as in Lax's work [5], where one considers deformations of operators $L$ in the equivalence class of operators of the form $U^{-1} L U$ for unitary $U$, here we consider deformations of operators $A$ in the equivalence class of operators $U_{1} A U_{2}$, where $U_{1}, U_{2}$ are two unitary operators. If we apply these ideas to formal differential operators we are led to the modified Korteweg-deVries equation which by $u=v^{\prime}$ $+v^{2}$ is transformed into (1.1). This follows from the fact that any deformation of $A$ of this kind gives rise to an isospectral deformation of $L$. The ideas are explained in Section 3.

On the other hand if $L=A^{*} A$ one gets a second differential operator $\tilde{L}=A A^{*}$ $=-D^{2}+\tilde{u}$ by exchanging the role of $A$ and $A^{*}$. Moreover, $\tilde{L}$ is also isospectrally deformed under the above deformation. This gives rise to a Bäcklund transformation of $u=v^{\prime}+v^{2}$ into $\tilde{u}=-v^{\prime}+v^{2}$ leaving the $X_{j}$ invariant. Applying this transformation repeatedly we construct the sequence $u_{d}$ of (1.3). This transformation has also been employed in the construction of $N$-soliton solutions $[11,12]$. In Section 4 we show that the $u_{d}$ so obtained actually represent the manifold $M_{d}$.

The above transformation $u \rightarrow \tilde{u}$ can be used to introduce a new eigenvalue into a differential operator without changing the rest of the spectrum (see [12]). We are dealing with the special case where the eigenvalue $\lambda=0$ is repeatedly introduced into the simple operator $L=-D^{2}$. Thus the resulting operators turn out to be reflectionless with continuous spectrum $[0, \infty)$ and a multiple eigenvalue at $\lambda=0$. For this interpretation one has to allow the potential $u_{d}$ to be complex so that the operator is no longer selfadjoint. In Section 5 we study the spectral properties of these operators which are quite simple as all eigenfunction representations are given explicitly in terms of elementary functions.

In [1] the functions $u_{d} \in M_{d}$ were described in terms of their poles $x_{1}, x_{2}, \ldots, x_{n}$, $n=\frac{1}{2}(d(d+1)$, which have to satisfy the conditions

$$
\sum_{\substack{j=1 \\ j \neq k}}^{n}\left(x_{k}-x_{j}\right)^{-3}=0 \text { for } k=1,2, \ldots, n .
$$

In contrast, here we give preference to the functions

$$
\theta_{d}=\prod_{j=1}^{n}\left(x-x_{j}\right)
$$


which are constructed in Section 2. As a consequence of their properties their roots satisfy (1.4) and all solutions of (1.4) can be so obtained. Thus the $\tau_{1}, \ldots, \tau_{d}$ can be viewed as uniformizing variables for the algebraic variety (1.4).

This paper is an extended and edited version of the MRC-report [19] where most of the results were presented.

The second author wishes to express his thanks for the hospitality of the University of Wisconsin. We are grateful to $\mathrm{C}$. Conley at whose seminar these results were presented and to $\mathrm{H}$. McKean for discussions on this subject. We also are indebted to P. Deift for pointing out the relevance of the factorization of second order operators.

\section{Construction of the Polynomials $\boldsymbol{\theta}_{\boldsymbol{k}}$}

a) A Recursion Formula

In this section we construct a sequence of polynomials $\theta_{k}\left(\tau_{1}, \ldots, \tau_{k}\right)$ for $k=0,1, \ldots$ of $k$ variables, which will be considered as polynomials of one variable $x=\tau_{1}$, the others figuring as parameters. As such they have the degree $n_{k}=\frac{1}{2} k(k+1)$. They are defined recursively by

$$
\theta_{0}=1, \theta_{1}=x=\tau_{1}
$$

and the dfifferential equation

$$
\theta_{k+1}^{\prime} \theta_{k-1}-\theta_{k+1} \theta_{k-1}^{\prime}=(2 k+1) \theta_{k}^{2}
$$

which leaves an integration constant available. We fix this constant by the normalization that the coefficient of $x^{n_{k-1}}$ in $\theta_{k+1}$ is equal to $\tau_{k+1}$. This defines the polynomials uniquely and at each recursive step one picks up a new integration constant $\tau$.

For the first few polynomials one finds

$$
\begin{aligned}
& \theta_{0}=1 \\
& \theta_{1}=x \\
& \theta_{2}=x^{3}+\tau_{2} \\
& \theta_{3}=x^{6}+5 \tau_{2} x^{3}+\tau_{3} x-5 \tau_{2}^{2} \\
& \theta_{4}=x^{10}+15 \tau_{2} x^{7}+7 \tau_{3} x^{5}-35 \tau_{2} \tau_{3} x^{2}+175 \tau_{2}^{3} x-\frac{7}{3} \tau_{3}^{2}+\tau_{4} x^{3}+\tau_{4} \tau_{2} .
\end{aligned}
$$

However, it is by no means obvious that the above differential equations can be solved within the class of polynomials. That this is the case will be shown below. In fact we will derive explicit formulae for these polynomials in terms of Wronskian determinants. For the Wronskian of two functions $A, B$ we use the notation

$$
[A, B]=A^{\prime} B-A B^{\prime} \text {. }
$$

\section{b) Factorization}

These polynomials are intimately related to potentials $u_{d}$ of the differential operators $-D^{2}+u_{d}$ obtained by repeated applications of the so-called Crum

2 Prime or $D$ stands for $\partial / \partial x$ 
transformation $^{3}$. In fact, in this way we will obtain an explicit representation of these polynomials.

To describe the Crum transformation we consider a second order differential operator

$$
L=-D^{2}+u ; D=\frac{d}{d x}
$$

and ask for a factorization $L-\lambda I=A^{*} A$ where $\lambda$ is a constant and

$$
A=D-v, A^{*}=-D-v
$$

are first order operators, which are formally adjoint to each other. The following construction is entirely algebraic and all operators are considered as formal differential operators without any boundary condition. For any differential operator $P$ of arbitrary finite order, having possibly complex valued coefficients we define $P^{*}$ as that operator for which

$$
\left(P^{*} f\right) \cdot g-f(P g)=\frac{d}{d x} Q(f, g)
$$

for arbitrary $C^{\infty}$-functions, with some polynomial $Q$ in $f, g$ and finitely many derivatives. For example, for $P=A$ we have

$$
\left(A^{*} f\right) \cdot g-f A g=-\frac{d}{d x}(f \cdot g) \text {. }
$$

To construct the most general such factorization we take $\phi \neq 0$ as a solution of the eigenvalue equation

$$
(L-\lambda) \phi=0
$$

and set

$$
A=\phi D \phi^{-1}, A^{*}=-\phi^{-1} D \phi .
$$

Then one verifies

$$
A^{*} A=L-\lambda \text {. }
$$

Indeed $A^{*} A$ is a second order differential operator, which is formally selfadjoint having $-D^{2}$ as leading term. Hence $A^{*} A$ is of the form $-D^{2}+q$. From the form of $A$ it is evident that $A^{*} A \phi=0$, i.e. $\left(-D^{2}+q\right) \phi=0$, hence by (2.3), (2.5),

$$
q=\frac{\phi^{\prime \prime}}{\phi}=u-\lambda \text {. }
$$

This verifies (2.7) which can of course also be done by direct calculation.

Thus every solution $\phi$ of (2.5) gives rise to such a factorization. Of course, $\phi$ and $c \phi$ give rise to the same factorization while two linearly independent solutions $\phi_{1}, \phi_{2}$ of (2.5) give actually different factorizations (2.7). Thus for every $\lambda$ we obtain

3 It may be more appropriate to refer to Burchenall and Chaundy [21] who studied such an operation already in 1923 and called it transference (see $\$ 3$ of [21]). The construction of so called Lax-pairs can be found also in this paper in $\$ 6$, and are called semicommutative operators. Although theirs is an entirely different motivation from the more recent developments it anticipates a remarkable number of results, in particular, the connection with hyperelliptic functions 
a one-parameter family of factorizations. These are the most general factorizations of the form (2.7).

The Crum transformation consists in mapping an operator $L=\lambda I+A^{*} A$ into the operator

$$
\tilde{L}=\lambda I+A A^{*}
$$

by just exchanging the role of $A$ and $A^{*}$. The operator $\tilde{L}$ is also of second order and of the form

$$
\tilde{L}=-D^{2}+\tilde{u} .
$$

One readily finds that

$$
\tilde{u}=u-2\left(\frac{\phi^{\prime}}{\phi}\right)^{\prime} \text {. }
$$

By the form of $\tilde{L}-\lambda=A A^{*}$ and $A^{*}=-\phi^{-1} D \phi$ it is clear that

$$
(\tilde{L}-\lambda) \phi^{-1}=0 \text {; }
$$

moreover, if one repeats the process and applies the Crum transformation to $\tilde{L}-\lambda$ with $\phi^{-1}$ in place of $\phi$ one is led back to $L-\lambda$.

Such a pair of operators

$$
L-\lambda=A^{*} A, \quad \tilde{L}-\lambda=A A^{*}
$$

have - with appropriate boundary conditions --very similar spectra--and this fact makes the operation essential for isospectral deformations. Indeed,

$$
(L-\mu) \psi=0
$$

implies

$$
(\tilde{L}-\mu) A \psi=0
$$

as is easily seen, and therefore $A$ maps eigenfunctions of $L$ into those of $\tilde{L}$, or into zero. For example, for $\mu=\lambda, \psi=\phi$ we have $A \phi=0$. This transformation is used to insert or remove one eigenvalue without changing the rest of the spectrum (see [12]).

With this motivation in mind we proceed to construct all potentials $u_{k}$ which can be obtained from $u_{0}=0$ by applying the above transformation $k$ times with the special choice $\lambda=0$. It is clear that we obtain functions $u_{k}\left(x, \tau_{1}, \ldots, \tau_{k}\right)$ depending on $k$ parameters. We show that the $u_{k}$ are closely related to the solutions $\theta_{k}$ of the recursion formula (2.2). Here we still assume the existence of these polynomials $\theta_{k}$; their construction will be given in c) of this section.

Lemma 1. If $\theta_{k}\left(x+\tau_{1}, \tau_{2}, \ldots, \tau_{k}\right)$ are solutions of (2.1) and (2.2) then the most general potential $u_{k}$ obtained by $k$ applications of the Crum transformation to $u_{0}=0$ with $\lambda=0$ is of the form

$$
u_{k}=-2\left(\log \theta_{k}\right)^{\prime \prime}, \quad k=0,1,2, \ldots
$$


Moreover, the eigenfunctions $\phi=\phi_{k}$ at the kth step are given by

$$
\phi_{k}=\frac{\theta_{k+1}}{\theta_{k}}, \quad L_{k} \phi_{k}=\left(-D^{2}+u_{k}\right) \phi_{k}=0 .
$$

Proof. For $k=0$ the definition $\theta_{0}=1$ gives $u_{0}=0$ and $-D^{2} \phi_{0}=0$ gives $\phi_{0}=a x+b$. We can assume $a \neq 0$ or even $a=1$, since $\phi_{0}=b$ gives nothing new, and thus $\phi_{0}=x$ $+b=\theta_{1}\left(x+\tau_{1}\right) / \theta_{0}$ if $b=\tau_{1}$.

In the following we will suppress the translation by $\tau_{1}$ and set $\tau_{1}=0$. Thus $\phi_{0}$ $=x=\theta_{1} / \theta_{0}$.

Now we proceed by induction and assume $\theta_{0}, \theta_{1}, \ldots, \theta_{k}$ have been found and

$$
L_{k}=-D^{2}+u_{k}=A_{k-1} A_{k-1}^{*}, A_{k-1}=\phi_{k-1} D \phi_{k-1}^{-1}
$$

are defined via (2.12), (2.13). In order to apply the Crum transformation to $L_{k}$ with $\lambda=0$ we need a solution $\phi$ of $L_{k} \phi=0$. From the above Equation (2.11) we see that $\left(\phi_{k-1}\right)^{-1}$ is such a solution; however, the Crum transformation applied with this function leads back to $L_{k-1}$ and gives nothing new. Therefore, we pick a solution $\phi$ $=\phi_{k}$ of $L_{k} \phi=0$ which is linearly independent of $\left(\phi_{k-1}\right)^{-1}$, i.e. one whose Wronskian with $\left(\phi_{k-1}\right)^{-1}$ is a nonvanishing constant. We normalize $\phi_{k}$ by setting

$$
\left[\phi_{k}, \phi_{k-1}^{-1}\right]=2 k+1 \text {. }
$$

Setting $\phi_{k}=\theta_{k+1} / \theta_{k}$ this relation agrees with the recursion formula (2.2).

It remains to verify $u_{k+1}=-2\left(\log \theta_{k+1}\right)^{\prime \prime}$ to complete the induction. By $(2.10)$ we have

$$
u_{k+1}=u_{k}-2\left(\log \phi_{k}\right)^{\prime \prime}=-2\left(\log \theta_{k}\right)^{\prime \prime}-2\left(\log \phi_{k}\right)^{\prime \prime}
$$

or $u_{k+1}=-2\left(\log \theta_{k} \phi_{k}\right)^{\prime \prime}=-2\left(\log \theta_{k+1}\right)^{\prime \prime}$ by (2.13), which completes the proof of Lemma 1.

We record some identities which follow from the above: Since $-\phi_{k}^{\prime \prime}+u_{k} \phi_{k}=0$ we find

$$
\begin{aligned}
\frac{\phi_{k}^{\prime \prime}}{\phi_{k}}-u_{k} & =\left\{\frac{\theta_{k+1}^{\prime \prime}}{\theta_{k+1}}-\frac{\theta_{k}^{\prime \prime}}{\theta_{k}}-2 \frac{\theta_{k}^{\prime}}{\theta_{k}}\left(\frac{\theta_{k+1}^{\prime}}{\theta_{k+1}}-\frac{\theta_{k}^{\prime}}{\theta_{k}}\right)\right\}+2 \frac{\theta_{k}^{\prime \prime}}{\theta_{k}}-2\left(\frac{\theta_{k}^{\prime}}{\theta_{k}}\right)^{2} \\
& =\frac{\theta_{k+1}^{\prime \prime}}{\theta_{k+1}}+\frac{\theta_{k}^{\prime \prime}}{\theta_{k}}-2 \frac{\theta_{k}^{\prime}}{\theta_{k}} \frac{\theta_{k+1}^{\prime}}{\theta_{k+1}}=0 .
\end{aligned}
$$

This can also be written as the identity

$$
\left[\theta_{k+1}^{\prime}, \theta_{k}\right]+\left[\theta_{k}^{\prime}, \theta_{k+1}\right]=0,
$$

which can also be derived from (2.1) and (2.2) directly. This relation is of second order but has the advantage of relating $\theta_{k}, \theta_{k+1}$ and not only $\theta_{k-1}, \theta_{k}, \theta_{k+1}$.

Finally, we remark, if we set $A_{k}=D-v_{k}$ we have

$$
v_{k}=\frac{\phi_{k}^{\prime}}{\phi_{k}} \text {. }
$$


Then we obtain

$$
\begin{aligned}
u_{k} & =v_{k}^{\prime}+v_{k}^{2} \\
u_{k+1} & =-v_{k}^{\prime}+v_{k}^{2} .
\end{aligned}
$$

Indeed, by (2.15) and (2.13)

$$
v_{k}^{\prime}+v_{k}^{2}=\frac{\phi_{k}^{\prime \prime}}{\phi_{k}}=u_{k}
$$

and

$$
-v_{k}^{\prime}+v_{k}^{2}=-2 v_{k}^{\prime}+u_{k}=-2\left(\frac{\phi_{k}^{\prime}}{\phi_{k}}\right)^{\prime}+u_{k}=u_{k+1}
$$

by (2.14).

\section{c) Explicit Representation of the $\theta_{k}$}

The factorization of $L_{k}$ gives rise to the formulae

$$
L_{k}=A_{k}^{*} A_{k}=A_{k-1} A_{k-1}^{*} \quad \text { for } \quad k=1,2, \ldots
$$

and

$$
L_{k} A_{k-1}=A_{k-1} L_{k-1}
$$

relating $L_{k}, L_{k-1}$. In order to get a direct relation between $L_{k}$ and $L_{0}=-D^{2}$ we introduce the operator.

$$
T_{k}=A_{k-1} A_{k-2} \ldots A_{1} A_{0}
$$

of order $k$. Then (2.18) yields

$$
L_{k} T_{k}=T_{k} L_{0}
$$

and (2.17) leads to the factorizations of $\left(L_{0}\right)^{k},\left(L_{k}\right)^{k}$ :

$$
L_{0}^{k}=T_{k}^{*} T_{k} ; \quad\left(L_{k}\right)^{k}=T_{k} T_{k}^{*} .
$$

It is interesting-and this was discovered by Crum [2]-that the operator $T_{k}$ can be expressed in terms of Wronskians: We define the Wronskian of $k$ functions $\psi_{1}, \psi_{2}, \ldots, \psi_{k}$ as

$$
W_{k}=W\left(\psi_{1}, \psi_{2}, \ldots, \psi_{k}\right)=\operatorname{det}\left(D^{i-1} \psi_{j}\right) \quad(i, j=1,2, \ldots, k) .
$$

For abbreviation we also set

$$
W_{k}(\chi)=W\left(\psi_{1}, \psi_{2}, \ldots, \psi_{k}, \chi\right)
$$

with another smooth function $\chi$. Then one has Jacobi's identity

$$
\left[W_{k}(\chi), W_{k+1}\right]=W_{k+1}(\chi) W_{k} \quad \text { for } \quad k=1,2, \ldots
$$

This is readily verified. The left-hand side is a linear differential operator of order $k+1$ in $\chi$ which clearly vanishes for $\chi=\psi_{1}, \psi_{2}, \ldots, \psi_{k}$ as well as for $\chi=\psi_{k+1}$. Thus, if we assume that the $\psi_{1}, \psi_{2}, \ldots, \psi_{k+1}$ are linearly independent, the left-hand side 
must be a multiple of $W_{k+1}(\chi)$. Comparing the highest coefficient one obtains (2.20).

We apply the above definitions to a system $\psi_{j}$ satisfying $\psi_{0}=0, \psi_{1}=x$ and

$$
\psi_{j}^{\prime \prime}=\psi_{j-1}, \quad j=1,2, \ldots, k .
$$

Then one verifies, for $\chi=1$ and setting $W_{0}=1$ that

$$
W_{k}(1)=(-1)^{k} W_{k-1} \quad \text { for } \quad k=1,2, \ldots
$$

To prove this we write

$$
W_{k}(1)=W\left(\psi_{1}, \psi_{2}, \ldots, \psi_{k}, 1\right)=(-1)^{k} W\left(1, \psi_{1}, \ldots, \psi_{k}\right)
$$

and since $\psi_{1}=x$ the last expression reduces by (2.21) to

$$
W_{k}(1)=(-1)^{k} W\left(\psi_{2}^{\prime \prime}, \psi_{3}^{\prime \prime}, \ldots, \psi_{k}^{\prime \prime}\right)=(-1)^{k} W\left(\psi_{1}, \psi_{2}, \ldots, \psi_{k-1}\right)=(-1)^{k} W_{k-1}
$$

proving (2.22).

Thus setting $\chi=1$ in (2.20) and using (2.22) we find

$$
\left[W_{k+1}, W_{k-1}\right]=W_{k}^{2} \text { for } k=1,2, \ldots
$$

and

$$
W_{0}=1, W_{1}=\psi_{1} .
$$

Thus with $\psi_{1}=x$ we see that $\theta_{k}$ and $W_{k}$ differ only by a multiplicative factor:

$$
\theta_{k}=\mu_{k} W_{k}
$$

which one determines to be

$$
\mu_{k}=1^{k} \cdot 3^{k-1} \cdot 5^{k-2} \ldots(2 k-1)^{1}=\prod_{j=1}^{k}(2 k-2 j+1)^{j} .
$$

But this factor is unessential for the following. The choice $\psi_{1}=x$ and (2.21) leads to

$$
\psi_{j}=\frac{x^{2 j-1}}{(2 j-1) !}+\sum_{i=0}^{j-2} \varrho_{j-i} \frac{x^{2 i}}{(2 i) !} .
$$

Equivalently one can define the $\psi_{j}$ by the generating function

$$
\sum_{j=1}^{\infty} \psi_{j} s^{2 j-1}=\sinh (s x)+\left(\sum_{i=2}^{\infty} \varrho_{i} s^{2 i-1}\right) \cosh (s x),
$$

where $\varrho_{2}, \varrho_{3}, \ldots$ are arbitrary parameters.

With this choice of $\psi_{1}, \psi_{2}, \ldots$ the Wronskians $W_{k}$ are polynomials of $x$ $=\varrho_{1}, \varrho_{2}, \ldots, \varrho_{k}$ with rational coefficients. It remains to compare these parameters $\varrho_{2}, \ldots, \varrho_{k}$ with the $\tau_{2}, \ldots, \tau_{k}$ in $\theta_{k}$. For this purpose we have to take account of the normalization of the $\theta_{k}$. If $\theta_{k+1}$ is a solution of (2.2), so is $\theta_{k+1}+c \theta_{k-1}$. The normalization of the $\theta_{k+1}$ can be expressed by

$$
\theta_{k+1}=\AA_{k+1}+\tau_{k+1} \theta_{k-1},
$$


where $\stackrel{\circ}{\theta+1}_{k+1}=\left.\theta_{k+1}\right|_{\tau_{k+1=0}}$. A similar formula holds for $W_{k+1}$ and $\stackrel{\circ}{W}_{k+1}$ $=\left.W_{k+1}\right|_{\varrho_{k+1}=0}$. Since $\psi_{k+1}=\stackrel{\circ}{\psi}_{k+1}+\varrho_{k+1}$ we have by $(2.22)$,

$$
\begin{aligned}
W_{k+1} & =\stackrel{\circ}{W}_{k+1}+\varrho_{k+1} W\left(\psi_{1}, \psi_{2}, \ldots, \psi_{k}, 1\right) \\
& =\stackrel{\circ}{W}_{k+1}+(-1)^{k} \varrho_{k+1} W_{k-1} .
\end{aligned}
$$

Comparing the last two equations and (2.24) we conclude

$$
\tau_{k+1}=\frac{\mu_{k+1}}{\mu_{k-1}}(-1)^{k} \varrho_{k+1}
$$

or

$$
\tau_{k}=\alpha_{k} \varrho_{k}, \quad \alpha_{k}=1^{2} \cdot 3^{2} \cdot 5^{2} \cdot \ldots(2 k-3)^{2} \cdot(2 k-1)(-1)^{k-1} .
$$

We summarize:

Lemma 2. The $\theta_{k}\left(\tau_{1}, \tau_{2}, \ldots, \tau_{k}\right)$ are polynomials of the $k$ variables with rational coefficients. Moreover, they have the homogeneity property

$$
\theta_{k}\left(\lambda \tau_{1}, \lambda^{3} \tau_{2}, \ldots, \lambda^{2 k-1} \tau_{k}\right)=\lambda^{n_{k}} \theta_{k}\left(\tau_{1}, \tau_{2}, \ldots, \tau_{k}\right)
$$

i.e. if we assign $\tau_{j}$ the weight $2 j-1$ then $\theta_{k}$ are "isobaric" of degree $n_{k}=k(k+1) / 2$.

It suffices to prove the additional remark.

If we replace $x, \tau_{j}, \theta_{k}$ by $x^{*}=\lambda x, \tau_{j}^{*}=\lambda^{2 j-1} \tau_{j}, \theta_{k}^{*}=\lambda^{n_{k}} \theta_{k}$ one verifies that (2.1), (2.2) and the normalization condition are preserved, hence by uniqueness $\theta_{k}^{*}$ $=\theta_{k}\left(\tau^{*}\right)$. This proves the statement.

\section{d) Parametrization}

Remark 1. The parameters $\tau_{2}, \tau_{3}, \ldots$ were introduced by a rather arbitrary normalization condition. One can free oneself from this arbitrariness by replacing the $\tau_{j}$ by

$$
\tau_{j}^{*}=a_{j} \tau_{j}+g_{j}\left(\tau_{2}, \tau_{3}, \ldots \tau_{j-1}\right),
$$

where $g_{j}$ are polynomials with rational coefficients and $a_{j} \neq 0$ is a rational number. Moreover, we require that $g_{j}$ be isobaric of degree $j$ which amounts to requiring that the transformation $\tau_{j} \rightarrow \tau_{j}^{*}$ commutes with $\tau_{j} \rightarrow \lambda^{2 j-1} \tau_{j}$. In fact, the above birational transformations form a group and we will reserve the freedom to pick an appropriate such transformation (see Section 4).

In the following we need another property of these polynomials.

Lemma 3. For fixed $d \geqq 1$ let

$$
\theta_{d}\left(x+\tau_{1}, \tau_{2}, \ldots, \tau_{d}\right)=x^{n}+\sigma_{1} x^{n-1}+\ldots+\sigma_{n}, \quad n=n_{d} .
$$

Then the $\sigma_{j}$ are isobaric polynomials in $\tau_{1}, \ldots, \tau_{d}$ of degree $j$. Moreover

$$
\sigma_{2 j-1}=\alpha_{j} \tau_{j}+q\left(\tau_{2}, \ldots, \tau_{j-1}\right) \text { for } j=1,2, \ldots, d,
$$

where $\alpha_{j} \neq 0$ and $q$ is a polynomial, isobaric of degree $2 j-1$.

Corollary. This lemma implies that the above relation can be solved for $\tau_{1}, \tau_{2}, \ldots, \tau_{d}$ and $\tau_{1}, \ldots, \tau_{d}$ expressed as isobaric polynomials with a nonvanishing linear term, of 
$\sigma_{1}, \sigma_{3}, \sigma_{5}, \ldots, \sigma_{2 d-1}$. Hence $\tau_{1}, \tau_{2}, \ldots, \tau_{d}$ are in birational, isobaric equivalence with $\sigma_{1}, \sigma_{3}, \ldots, \sigma_{2 d-1}$.

Proof. It is obvious from the above proposition that the $\sigma_{j}$ are isobaric polynomials in $\tau_{2}, \ldots, \tau_{d}$ and we just have to verify that $\alpha_{j} \neq 0$. Of course, $\alpha_{j}=\alpha_{j}(d)$ depends on $d$, and we simply compute it.

Clearly $\alpha_{j}$ is the coefficient of $\tau_{j} x^{n-2 j+1}$ in $\theta=\theta_{d}$ and therefore we have

$$
\frac{\partial}{\partial \tau_{j}} \theta=\alpha_{j} x^{n-2 j+1} \text { for } \tau_{1}=0, \ldots, \tau_{d}=0
$$

while

$$
\theta=x^{n} \text { for } \tau_{1}=0, \ldots, \tau_{d}=0 .
$$

Hence if we differentiate (2.2) with respect to $\tau_{j}, \partial / \partial \tau_{j}$ denoted by a dot, $\partial / \partial x$ by a prime, we find

$$
\left(\dot{\theta}_{d+1}^{\prime} \theta_{d-1}-\dot{\theta}_{d+1} \theta_{d-1}^{\prime}\right)+\left(\theta_{d+1}^{\prime} \dot{\theta}_{d-1}-\theta_{d+1} \dot{\theta}_{d-1}^{\prime}\right)=2(2 d+1) \theta_{d} \dot{\theta}_{d} .
$$

For $\tau_{1}=\tau_{2}=\ldots=\tau_{d}=0$ this gives

$$
\begin{aligned}
& \left\{\left(n_{d+1}-2 j+1\right)-n_{d-1}\right\} \alpha_{j}(d+1)+\left\{n_{d+1}-\left(n_{d-1}-2 j+1\right)\right\} \alpha_{j}(d-1) \\
& \quad=2(2 d+1) \alpha_{j}(d)
\end{aligned}
$$

or since $n_{d+1}-n_{d-1}=2 d+1$

$$
(d-j+1) \alpha_{j}(d+1)+(d+j) \alpha_{j}(d-1)=(2 d+1) \alpha_{j}(d) .
$$

This recursion formula for $\alpha_{j}(d)$, together with $\alpha_{j}(d)=1$ for $d=j$ (by normalization) and $\alpha_{j}(d)=0$ for $d>j$, determines $\alpha_{j}(d)$ uniquely. In fact one finds explicitly

$$
\alpha_{j}(d)=\left(\begin{array}{c}
d+j \\
d-j
\end{array}\right)=\left(\begin{array}{c}
d+j \\
2 j
\end{array}\right) \neq 0 \text { for } j=1,2, \ldots, d
$$

which proves the lemma.

This lemma has the following consequence: The polynomials $\theta_{d}\left(x+\tau_{1}\right.$, $\left.\tau_{2}, \ldots, \tau_{d}\right)$ are uniquely determined by the choise of the $\tau_{1}, \ldots, \tau_{d}$. Indeed, if

$$
\theta_{d}\left(x+\tau_{1}, \tau_{2}, \ldots, \tau_{d}\right)=\theta_{d}\left(x+\hat{\tau}_{1}, \hat{\tau}_{2}, \ldots, \hat{\tau}_{d}\right)
$$

for all $x$ then the coefficients $\sigma_{j}(\tau)=\sigma_{j}(\hat{\tau})$ agree, which implies by the above lemma that $\tau_{k}=\hat{\tau}_{k}$. This remark implies that the representation (1.3) of $u \in M_{d}$ is one to one.

Remark 2. For $\tau_{2}=\tau_{3}=\ldots=\tau_{d-2}=0$ one can easily compute $\theta_{d}$ explicitly and find for $d \geqq 3$

$$
\theta_{d}=x^{n_{d-4}}\left\{x^{4 d-6}+(2 d-1) \tau_{d-1} x^{2 d-3}-\frac{2 d-1}{2 d-5} \tau_{d-1}^{2}+\tau_{d} x^{2 d-5}\right\} .
$$

This formula contains as special cases those of [1], Propositions 2 and 3, Section 5.

e) Representation $T_{k}$

We conclude this section with an explicit representation of $T_{k}$ due to Crum [2]. 
Lemma 4. The mapping

$$
\chi \rightarrow T_{k} \chi=A_{k-1} A_{k-2} \ldots A_{0} \chi
$$

can be represented in the form

$$
T_{k} \chi=\frac{W_{k}(\chi)}{W_{k}} \quad \text { for } \quad k=1,2, \ldots
$$

Proof. This formula clearly holds for $k=1$ and we verify it by induction. It suffices to check

$$
A_{k} T_{k} \chi=A_{k} \frac{W_{k}(\chi)}{W_{k}}=\frac{W_{k+1}(\chi)}{W_{k+1}}=T_{k+1} \chi .
$$

Indeed, with $A_{k}=\phi_{k} D \phi_{k}^{-1} ; \phi_{k}=W_{k+1} / W_{k}$

$$
A_{k} \frac{W_{k}(\chi)}{W_{k}}=\phi_{k} D\left(\frac{W_{k}}{W_{k+1}} \frac{W_{k}(\chi)}{W_{k}}\right)=\phi_{k} \frac{\left[W_{k}(\chi), W_{k+1}\right]}{W_{k+1}^{2}}
$$

Using (2.20) the right-hand side becomes

$$
\phi_{k} \frac{W_{k+1}(\chi) \cdot W_{k}}{W_{k+1}^{2}}=\frac{W_{k+1}(\chi)}{W_{k+1}},
$$

as we wanted to show.

\section{A Deformation Problem for First Order Differential Operators}

In [5] Lax related the Korteweg-deVries equation-as well as its higher analogues - to the isospectral deformation of the operator

$$
L=-D^{2}+u \text {, }
$$

where $u=u(x)$ is a $C^{\infty}$-function. We will describe an analogue problem for the first order operator

$$
A=D-v
$$

with $v=v(x)$.

For motivation of the following we consider at first a bounded linear operator $A$ in a Hilbert space and call $A$ equivalent to $A_{0}$ if there exist two unitary operators $U_{1}, U_{2}$ such that

$$
U_{1}^{-1} A U_{2}=A_{0} \text {. }
$$

Clearly the invariants of this equivalence relation are the spectral invariants of $A^{*} A$ and of $A A^{*}$.

We ask for deformations $A(t)$ of $A_{0}=A(0)$ which remain in the same equivalence class. Assuming that $U_{1}=U_{1}(t), U_{2}=U_{2}(t)$ are defined through differential equations

$$
\dot{U}_{j}=B_{j} U_{j} ; \quad U_{j}(0)=I ; \quad j=1,2
$$


with skew Hermitian $B_{j}$, we obtain by differentiation of (3.3)

$$
U_{1}^{-1}\left(\dot{A}-B_{1} A+A B_{2}\right) U_{2}=0
$$

or

$$
\dot{A}=B_{1} A-A B_{2} .
$$

We apply this consideration to $A=D-v, v=v(x, t)$ and choose

$$
B_{j}=D^{3}+b_{j} D+D b_{j}
$$

as skew Hermitian operator. We now consider (3.4) as a formal relation for differential operators. The left-hand side of (3.4) is a multiplication operator, namely multiplication by $-v_{t}$ and $b_{1}, b_{2}$ have to be so determined that in $B_{1} A$ $-A B_{2}$ the coefficients of $D^{4}, D^{3}, D^{2}, D$ vanish. The first two coefficients vanish automatically while the other two are

$$
\begin{aligned}
& +3 v^{\prime}+2\left(b_{1}-b_{2}\right) \\
& -3 v^{\prime \prime}+b_{1}^{\prime}-3 b_{2}^{\prime}-2\left(b_{1}-b_{2}\right) v .
\end{aligned}
$$

Setting these expressions equal to zero yields two linear equations for $b_{1}, b_{2}$ with the solution

$$
\begin{aligned}
& b_{1}=-\frac{3}{4}\left(-v^{\prime}+v^{2}\right)+c \\
& b_{2}=-\frac{3}{4}\left(v+v^{2}\right)+c
\end{aligned}
$$

with an arbitrary integration constant $c$. This constant reflects the trivial solution of (3.4) with $B_{j}=2 c D, v_{t}=2 c v_{x}$. Therefore we set $c=0$ and obtain from (3.4) a partial differential equation for $v$ which is computed to be

$$
v_{t}=\frac{1}{4} v^{\prime \prime \prime}-\frac{3}{2} v^{2} v^{\prime} .
$$

This is the so-called modified Korteweg-deVries equation which was used by Miura [7] in his derivation of the conservation laws for the $K d V$ equation. For this purpose he showed that the function

$$
u=v^{2}+v^{\prime}
$$

satisfies

$$
u_{t}=\frac{1}{4} u^{\prime \prime \prime}-\frac{3}{2} u u^{\prime},
$$

if $v$ is a solution of (3.5). This remarkable fact has a natural explanation in the following observation:

If $u=v^{\prime}+v^{2}$ then the operator (3.1) can be factored as

$$
L=A^{*} A \text {, }
$$

where

$$
A^{*}=-D-v
$$


is the formal adjoint of $A$. Moreover, the deformation equation (3.4) gives rise to a deformation equation for $L$

$$
\dot{L}=\dot{A}^{*} A+A^{*} \dot{A}=-A^{*} B_{1} A+B_{2} A^{*} A+A^{*} B_{1} A-A^{*} A B_{2}=\left[B_{2}, L\right] ;
$$

here we used that $B_{j}^{*}=-B_{j}, B_{j}=B_{j}(v)$. This is precisely the deformation problem

$$
\dot{L}=[B, L] ; B=B(u)=D^{3}+(b(u) D+D b(u))
$$

studied by Lax [5], which leads to (3.7) and $B_{2}(v)=B\left(v^{\prime}+v^{2}\right)$, provided the arbitrary constant is normalized appropriately. This shows then that any solutions $v$ of (3.5) give rise to a solution $u$ of (3.7) via (3.6). This appears as a consequence of the factorization (3.8).

If instead we consider the operator

$$
\tilde{L}=A A^{*}=-D+\tilde{u}
$$

with $\tilde{u}=-v^{\prime}+v^{2}$, then clearly we find analogously to (3.9)

$$
\dot{\tilde{L}}=\left[B_{1}, \tilde{L}\right]
$$

and $B_{1}(v)=B(\tilde{u})=B\left(-v^{\prime}+v^{2}\right)$, where $B(\tilde{u})$ again denotes the third order operator obtained by Lax. Moreover, $\tilde{u}=-v^{\prime}+v^{2}$ is automatically a solution of (3.7). The duality map $u \rightarrow \tilde{u}$ which takes solutions of (3.7) again into solutions of the same equation, a so-called Bäcklund transformation, is here related to the deformation of two products $A A^{*}, A^{*} A$, i.e. to exchanging $A$ and $A^{*}$.

These considerations can be generalized to the higher Korteweg-deVries operators $X_{j}$ by considering real skew Hermitian operators $B_{1}, B_{2}$ of degree $2 j-1$. This leads for each $j=1,2, \ldots$ to two skew symmetric differential operators

$$
\left\{\begin{array}{l}
B_{1}(v)=D^{2 j-1}+\sum_{s=1}^{j-1}\left(b_{1, s} D^{2 s-1}+D^{2 s-1} b_{1, s}\right) \\
B_{2}(v)=D^{2 j-1}+\sum_{s=1}^{j-1}\left(b_{2, s} D^{2 s-1}+D^{2 s-1} b_{2, s}\right),
\end{array}\right.
$$

where $b_{1, s}, b_{2, s}$ are polynomials of $v, v^{\prime}, \ldots$ and such that $B_{1} A-A B_{2}$ is a multiplication operator. One can make $B_{1}, B_{2}$ unique by requiring $B_{1}, B_{2}$ to be isobaric of weight $2 j-1$ where $D$ and $v$ are assigned the weight 1 , hence $v^{(s)}=D^{s} v$ the weight $s+1$. The proof of uniqueness can easily be given by comparing coefficients of $D^{\alpha}$ in (3.4) and will be omitted.

We will show now that these operators $B_{1}, B_{2}$ are closely related to the operator occurring in the Lax formalism. We recall (see, e.g. $[5,6]$ ) that for each $j$ $=1,2, \ldots$ there exists a unique skew symmetric differential operator

$$
B=B(u)=D^{2 j-1}+\sum_{s=1}^{j-1}\left(\beta_{s} D^{2 s-1}+D^{2 s-1} \beta_{s}\right)
$$

with $\beta_{s}$ being polynomials of $u, u^{\prime}, \ldots$ such that $[B, L]=B L-L B$ is a multiplication operator and $B$ is isobaric of weight $2 j-1$, if $u$ is assigned the weight 2 and $D$ the weight 1 . The relation of $B_{1}, B_{2}$ of (3.10) to the operator $B$ is expressed by: 
Theorem 1. The unique isobaric differential operators $B_{1}, B_{2}$ for which $B_{1} A-A B_{2}$ is a multiplication operator are given by

$$
\begin{aligned}
& B_{1}(v)=B\left(-v^{\prime}+v^{2}\right) \\
& B_{2}(v)=B\left(v^{\prime}+v^{2}\right) .
\end{aligned}
$$

Proof. For a given $v$ we set $A=D-v$

$$
\begin{array}{ll}
L=A^{*} A=-D^{2}+u, & u=v^{\prime}+v^{2} \\
\tilde{L}=A A^{*}=-D^{2}+\tilde{u}, & \tilde{u}=-v^{\prime}+v^{2}
\end{array}
$$

and construct the isobaric differential operators $B=B(u), \tilde{B}=B(\tilde{u})$ of the form (3.11) for which $[B, L],[\tilde{B}, \tilde{L}]$ are multiplication operators. We define $B_{1}(v)$ $=B\left(-v^{\prime}+v^{2}\right)=B(\tilde{u}), B_{2}(v)=B\left(v^{\prime}+v^{2}\right)=B(u)$ and note that $B_{1}, B_{2}$ are clearly isobaric. Next we verify that $Q=B_{1} A-A B_{2}$ is a multiplication operator. For this purpose we show first that the operators

$$
\begin{aligned}
& A^{*} Q+Q^{*} A=X(u) \\
& Q A^{*}+A Q^{*}=\tilde{X}(u)
\end{aligned}
$$

are both multiplication operators. Indeed, since $B_{i}^{*}=-B_{i}$ for $i=1,2$ we have

$$
A^{*} Q+Q^{*} A=A^{*}\left(B_{1} A-A B_{2}\right)+\left(A^{*} B_{1}^{*}-B_{2}^{*} A^{*}\right) A=\left[B_{2}, A^{*} A\right]=\left[B_{2}, L\right]
$$

which by construction is a multiplication operator. Similarly

$$
Q A^{*}+A Q^{*}=\left[B_{1}, A A^{*}\right]=[\tilde{B}, \tilde{L}] .
$$

From the above two relations (3.12) we conclude that

$$
Q=q_{1} D^{r}+q_{2} D^{r-1}+\ldots, \quad 0 \leqq r<2 j
$$

is actually a multiplication operator. Otherwise, we would have $r \geqq 1, \quad q_{1} \neq 0$. Computing the highest order terms in (3.12) we find

$$
A^{*} Q+Q^{*} A=\left(-1+(-1)^{r}\right) q_{1} D^{r+1}+\ldots
$$

Hence, if $r \geqq 1$ we must have $r$ even. For even $r$ we compute the terms of next order as

$$
\begin{aligned}
& A^{*} Q+Q^{*} A=\left\{(r-1) q_{1}^{\prime}-2\left(v q_{1}+q_{2}\right)\right\} D^{r}+\ldots \\
& Q A^{*}+A Q^{*}=\left\{(r+1) q_{1}^{\prime}-2\left(v q_{1}+q_{2}\right)\right\} D^{r}+\ldots
\end{aligned}
$$

and for $r \geqq 2$ we conclude that the coefficients of $D^{r}$ must vanish, hence $q_{1}^{\prime}=0$, i.e. $q_{1}$ is independent of $x$. On the other hand, $Q$ is isobaric of weight $2 j-1+1=2 j$, i.e. $q_{1}$ is of weight $2 j-t>0$, hence $q_{1}=0$. The last equation implies that $q_{2}=0$ also. consequently that $r=0$, i.e. $Q$ is a multiplication operator. By the uniqueness of $B_{1}, B_{2}$ the theorem is proven.

For fixed $j$ we introduce the multiplication operators

$$
[B(u), L]=X_{j}(u), \quad-\left(B_{1}(v) A-A B_{2}(v)\right)=Y_{j}(v)
$$


and accordingly arrive from $\dot{L}=[B, L], \dot{A}=B_{1} A-A B_{2}$ at the differential equations

$$
\begin{aligned}
& \frac{\partial u}{\partial t}=X_{j}(u) \\
& \frac{\partial v}{\partial t}=Y_{j}(v) .
\end{aligned}
$$

The first is the family of higher order Korteweg-deVries equations while the $Y_{j}(v)$ are generalizations of the modified Korteweg-deVries equation.

As a consequence of Theorem 1 we note that the transformations $u=v^{\prime}+v^{2}, \tilde{u}$ $=-v^{\prime}+v^{2}$ take one equation into the other, i.e. we have the transformation formulae

$$
\left\{\begin{array}{l}
X_{j}\left(v^{\prime}+v^{2}\right)=(D+2 v) Y_{j}(v) \\
X_{j}\left(-v^{\prime}+v^{2}\right)=(-D+2 v) Y_{j}(v) .
\end{array}\right.
$$

Clearly, by its derivation $X_{j}, Y_{j}$ are isobaric of weight $2 j+1,2 j$ respectively. Finally, we need that

$$
Y_{j}(-v)=-Y_{j}(v)
$$

is odd. This follows at once from the fact that the adjoint of

$$
Q(v)=B_{1} A-A B_{2}=-Y_{j}(v)
$$

on one hand, as a multiplication operator, agrees with $Q$, on the other hand is

$$
Q^{*}(v)=A^{*} B_{1}^{*}-B_{2}^{*} A^{*}=-A^{*} B_{1}+B_{2} A^{*},
$$

and since $A^{*}(v)=-D-v=-A(-v)$, and $B_{1}(v)=B_{2}(-v)$ by Theorem 1 , we conclude that

$$
Q^{*}(v)=-Q(-v)
$$

Hence $Q(v)=-Q(-v)$, proving (3.14).

Finally we conclude from (3.13): Since the $X_{j}$ commute, i.e. $X_{j} X_{k}=X_{k} X_{j}$ also the $Y_{j}$ commute.

The above facts are - at least for $j=2$ - not new but we wanted to derive them in generality to show the natural implication of the factorization process and also wished to make the following proof selfcontained.

We mention in passing that the $X_{j}(u)$ and the $Y_{j}(v)$ can be defined recursively. According to Lenard (see [3]) the $X_{j}$ satisfy

$$
X_{j+1}(u)=\left(u D+D u-\frac{1}{2} D^{3}\right) Z_{j} \quad \text { where } \quad c_{j} X_{j}=D Z_{j}
$$

defines $Z_{j}$, where the $c_{j}$ are constants. With $X_{1}(u)=u^{\prime}$ this recursion formula can be used to define the $X_{j}(u)$ and they turn out to be the same as defined above if the constants $c_{j} \neq 0$ are chosen appropriately. This follows from the formulae in [6].

Formally, the recursion formula can be written as

$$
X_{j+1}=c_{j} R X_{j} \text { where } R=u+D u D^{-1}-\frac{1}{2} D^{2} .
$$


It is easy to derive an analogous recursion formula for the $Y_{j}(v)$ if one uses (3.13). One finds

$$
Y_{j+1}=c_{j} S Y_{j}, \quad Y_{1}=v^{\prime},
$$

where

$$
S(v)=2 v^{2}+2 v^{\prime} D^{-1} v-\frac{1}{2} D^{2} .
$$

This expression $S$ was determined so that the identity

$$
(2 v+D) S(v)=R\left(v^{\prime}+v^{2}\right)(2 v+D)
$$

holds, which makes evident that (3.15) goes into (3.16) under the transformation $u$ $=v^{\prime}+v^{2}$. The recursion formula (3.16) is due to Olver [10]. Incidentally, one can use the evenness of $S$ to derive (3.14) inductively.

In the following we will not need this recursion formula, however, and work with Theorem 1, (3.13) and (3.14).

\section{The Invariant Manifold $M_{d}$}

In this section we show that the potentials

$$
u_{d}=-2 D^{2}\left(\log \theta_{d}\right)
$$

constructed with the polynomials $\theta_{d}$ of Section 2 define the manifold $M_{d}$ of the introduction. We recall that $M_{d}$ was defined as the orbit of $\exp \left(\sum_{j} t_{j} X_{j}\right)$ through the element $u=d(d+1) x^{-2}$ which is a connected $d$-dimensional manifold. Since for $\tau_{1}=\tau_{2}=\ldots=\tau_{d}=0$ we have $\theta_{d}=x^{n_{d}}, u_{d}=2 n_{d} x^{-2}$ it will suffice to show that the $d$ dimensional family of functions (4.0) also are left invariant under the flows $\exp \left(\sum t_{j} X_{j}\right)$. This is the content of the following theorem.

Theorem 2. There is a unique choice of rational functions $\gamma_{k j}\left(\tau_{2}, \ldots, \tau_{j}\right)$ and differential operators

$$
\Gamma_{k}=\sum_{j=1}^{\infty} \gamma_{k j} \frac{\partial}{\partial \tau_{j}}
$$

such that for $d=0,1,2, \ldots$

$$
X_{k}\left(u_{d}\right)=\Gamma_{k} u_{d}
$$

and

$$
Y_{k}\left(v_{d}\right)=\Gamma_{k} v_{d} \quad \text { where } \quad v_{d}=\frac{\theta_{d+1}^{\prime}}{\theta_{d+1}}-\frac{\theta_{d}^{\prime}}{\theta_{d}} .
$$

(Since $u_{d}, v_{d}$ depend only on finitely many variables the sum breaks off.)

In other words, if the $\tau_{j}$ satisfy

$\frac{d \tau_{j}}{d t_{k}}=\gamma_{k j}\left(\tau_{2}, \ldots, \tau_{j}\right), \quad j \leqq d$, 
then $u=u_{d}\left(x+\tau_{1}, \tau_{2}, \ldots, \tau_{d}\right)$ solves the equation

$$
\frac{\partial u}{\partial t_{k}}=X_{k}(u) \text {. }
$$

Proof. We proceed by induction on $d$. For $d=0$ we have $u_{0}=0$ and therefore $X_{k}\left(u_{0}\right)$ $=0$. Assume next that $\gamma_{k j}=\gamma_{k j}\left(\tau_{2}, \ldots, \tau_{j}\right)$ for $j=1,2, \ldots, d$ have been determined such that (4.1) holds. Then we conclude from (3.13) and $u_{d}=v_{d}^{\prime}+v_{d}^{2}$ with

$$
\begin{aligned}
& v_{d}=\phi_{d}^{\prime} / \phi_{d}, \phi_{d}=\theta_{d+1} / \theta_{d}, \Gamma_{k}^{0}=\left.\Gamma_{k}\right|_{\gamma_{k, d+1}=0} \\
& \left(2 v_{d}+D\right) Y_{k}\left(v_{d}\right)=X_{k}\left(u_{d}\right)=\Gamma_{k}^{0} u_{d}=\left(2 v_{d}+D\right) \Gamma_{k} v_{d}
\end{aligned}
$$

or

$$
\left(2 v_{d}+D\right)\left(Y_{k}\left(v_{d}\right)-\Gamma_{k}^{0} v_{d}\right)=0 .
$$

Since $\psi=\phi_{d}^{-2}$ is a solution of $\left(2 v_{d}+D\right) \psi=0$ we conclude that

$$
Y_{k}\left(v_{d}\right)-\Gamma_{k}^{0} v_{d}=c \phi_{d}^{-2}
$$

with $c$ being a rational function of $\tau_{2}, \ldots, \tau_{d+1}$. On the other hand $v_{d}$ depends on $\tau_{d+i}$ and in

$$
\Gamma_{k} v_{d}=\sum_{j \leqq d} \gamma_{k j} \frac{\partial v_{d}}{\partial \tau_{j}}+\gamma_{k, d+1} \cdot \frac{\partial v_{d}}{\partial \tau_{d+1}}=\Gamma_{k}^{0} v_{d}+\gamma_{k, d+1} \frac{\partial v_{d}}{\partial \tau_{d+1}},
$$

the coefficient $\gamma_{k, d+1}$ can be uniquely determined so that $c=0$, if $\Gamma_{k}^{0}$ is replaced by $\Gamma_{k}$ in (4.3). Indeed, $v_{d}=\phi_{d}^{\prime} / \phi_{d}=\theta_{d+1}^{\prime} / \theta_{d+1}-\theta_{d}^{\prime} / \theta_{d}$ where $\theta_{d}$ is independent of $\tau=\tau_{d+1}$ while $d \theta_{d+1} / d \tau=\theta_{d-1}$. Hence, by (2.2)

$$
\frac{\partial v_{d}}{\partial \tau_{d+1}}=\left(\frac{\theta_{d-1}}{\theta_{d+1}}\right)^{\prime}=-(2 d+1) \frac{\theta_{d}^{2}}{\theta_{d+1}^{2}}=-(2 d+1) \phi_{d}^{-2}
$$

and the coefficient of $\gamma_{k, d+1}$ in (4.4) is $-(2 d+1) \phi_{d}^{-2}$; thus we have from (4.3)

$$
\phi_{d}^{2}\left\{Y_{k}\left(v_{d}\right)-\Gamma_{k} v_{d}\right\}=(2 d+1) \gamma_{k, d+1}+c .
$$

Thus, if we set $\gamma_{k, d+1}=-(2 d+1)^{-1} c$ we obtain

$$
Y_{k}\left(v_{d}\right)=\Gamma_{k} v_{d}
$$

as claimed in (4.2). This determines $\gamma_{k, d+1}=\gamma_{k, d+1}\left(\tau_{2}, \ldots, \tau_{d+1}\right)$ uniquely.

Using that $Y_{k}(-v)=-Y_{k}(v)$ we conclude from (3.13)

$$
\begin{aligned}
X_{k}\left(u_{d+1}\right) & =X_{k}\left(-v_{d}^{\prime}+v_{d}^{2}\right)=\left(-2 v_{d}+D\right) Y_{k}\left(-v_{d}\right) \\
& =\left(2 v_{d}-D\right) Y_{k}\left(v_{d}\right) \\
& =\left(2 v_{d}-D\right) \Gamma_{k} v_{d} \\
& =\Gamma_{k}\left(v_{d}^{2}-v_{d}^{\prime}\right)=\Gamma_{k} u_{d+1}
\end{aligned}
$$

which completes the induction and the proof.

Lemma 5. The $\gamma_{k j}=\gamma_{k j}(\tau)$ are polynomials of $\tau_{2}, \tau_{3}, \ldots$ with rational coefficients. If we assign $\tau_{m}$ the weight $2 m-1$ the $\gamma_{k j}$ are isobaric of weight $2(j-k)$. In particular, they depend on $\tau_{l}$ with $l \leqq[j-k] \leqq j-1$ only. Moreover, $\gamma_{k k}$ is a nonvanishing constant. 
Proof. First we express the differential equation $u_{t}=X_{k}(u)$ via $u=-2(\log \theta)^{\prime \prime}$ in terms of

$$
\theta=\theta_{d}=x^{n}+\sigma_{1} x^{n-1}+\ldots+\sigma_{n}
$$

with $n=n_{d}$. This expresses the differential equation in terms of the $\sigma_{j}$ which finally are transferred to the $\tau_{j}$ via the corollary of Lemma 3 of Section 2.

From

$$
-2 \frac{\partial}{\partial t} D\left(\frac{\theta^{\prime}}{\theta}\right)=u_{t}=X_{k}(u)=D \frac{\delta H_{k}}{\delta u}
$$

we conclude that

$$
\theta^{-2}\left(\dot{\theta} \theta^{\prime}-\dot{\theta}^{\prime} \theta\right)=\frac{1}{2} \frac{\delta H_{k}}{\delta u},
$$

an integration constant being eliminated by the isobaric property. The right-hand side depends on $u, u^{\prime}, \ldots$, and we observe that by (4.0)

$$
D^{m} u=\theta^{-m-2} P_{m}\left(\theta, \theta^{\prime}, \ldots\right)
$$

with $P_{m}$ being a polynomial in $\theta, \theta^{\prime}, \ldots$ Since $\delta H_{k} / \delta u$ is isobaric of degree $2 k$ we find that

$$
\theta^{2 k} \frac{1}{2} \frac{\delta H_{k}}{\delta u}=Q\left(\theta, \theta^{\prime}, \ldots\right)
$$

is a polynomial in $\theta, \theta^{\prime}, \ldots$. Thus the differential equation takes the form

$$
\theta^{2 k-2}\left(\dot{\theta} \theta^{\prime}-\dot{\theta}^{\prime} \theta\right)=Q\left(\theta, \theta^{\prime}, \ldots\right) \text {. }
$$

In this equation we compare coefficients of $x$. The term of highest power in $x$ containing $\dot{\sigma}_{j}$ is

$$
x^{(2 k-2) n} x^{n-j} x^{n-1}(n-(n-j)) \dot{\sigma}_{j}=x^{2 k n-j-1} j \dot{\sigma}_{j}
$$

i.e. comparing the coefficients of $x^{2 k n-j-1}$ we find

$$
j \dot{\sigma}_{j}=\sum_{m<j} a_{m} \dot{\sigma}_{m}+a_{j},
$$

where $a_{1}, a_{2}, \ldots, a_{j}$ are polynomials in the $\sigma$. Thus we find

$$
\dot{\sigma}_{j}=b_{j}\left(\sigma_{1}, \ldots, \sigma_{n}\right)
$$

with polynomials $b_{j}$. Finally using the corollary of Lemma 3 we express these differential equations in terms of $\tau_{1}, \tau_{2}, \ldots$ in the form

$$
\dot{\tau}_{j}=\gamma_{k j}\left(\tau_{1}, \tau_{2}, \ldots\right) \text {. }
$$

One readily checks the homogeneity of the $\gamma_{k j}$ to be given by

$$
\gamma_{k j}\left(\lambda \tau_{1}, \lambda^{3} \tau_{2}, \ldots\right)=\lambda^{2 j-2 k_{\gamma_{j j}}}
$$


by using an argument like in the proof of Lemma 2, and observing the isobaric property of $X_{k}$. In particular, $\gamma_{k j}=0$ for $j<k$ and $\gamma_{k k}$ is a constant.

To evaluate this constant we use the fact that

$$
u_{d}=\frac{2 n_{d}}{x^{2}} \quad \text { for } \quad \tau_{2}=\tau_{3}=\ldots=\tau_{d}=0
$$

and for $d=k$

$$
X_{k} u_{k}=c_{k} x^{-(2 k+1)}
$$

with a rational constant $c_{k} \neq 0$ as one computes from (3.15). Thus we have for $\tau_{2}$ $=\tau_{3}=\ldots=\tau_{k}=0$

$$
0 \neq X_{k} u_{k}=\Gamma_{k} u_{k}=\sum_{j=1}^{k} \frac{\partial u_{k}}{\partial \tau_{j}} \gamma_{k j}=\frac{\partial u_{k}}{\partial \tau_{k}} \gamma_{k k},
$$

as $\gamma_{k j}=0$ for $j<k$. Hence $\gamma_{k k} \neq 0$ as was claimed. by

Thus the differential equations induced by $X_{k}$ are given by $\Gamma_{k}$, or equivalently

$$
\dot{\tau}_{j}=\gamma_{k j}\left(\tau_{2}, \tau_{3}, \ldots, \tau_{j-1}\right),
$$

with isobaric polynomials $\gamma_{k j}$. Obviously the equations can be solved recursively as polynomials in $t$. In fact, more is true: There exists an isobaric birational transformation $\tau_{j} \rightarrow \tau_{j}^{*}$ such that for all $k \geqq 1$ we have

$$
\Gamma_{k}=\frac{\partial}{\partial \tau_{k}^{*}}
$$

i.e. the above differential equation reduces to

$$
\Gamma_{k} \tau \tau_{j}^{*}=\delta_{k j}
$$

and $\tau_{k}^{*}$ can be interpreted as the time variable for the flow $X_{k}$. This is a simple consequence of the fact that the $X_{k}$ - and hence the $\Gamma_{k}$-commute. We recall that the $\tau_{k}$ were introduced by an artificial normalization and these parameters are actually defined only up to the group of birational transformations which commute with $\tau_{j} \rightarrow \lambda^{2 j-1} \tau_{j}$. Now we make the unique choice of the parameters so that they are adapted to the $\mathrm{KdV}$ flows.

Theorem 3. There exists a unique birational transformation

$$
\tau_{j}^{*}=a_{j} \tau_{j}+g_{j}\left(\tau_{2}, \tau_{3}, \ldots, \tau_{j-1}\right)
$$

with $g_{j}$ being isobaric polynomials

$$
g_{j}\left(\lambda^{3} \tau_{2}, \lambda^{5} \tau_{3}, \ldots, \lambda^{2 j-3} \tau_{j-1}\right)=\lambda^{2 j-1} g_{j}\left(\tau_{2}, \ldots, \tau_{j-1}\right)
$$

with rational coefficients and a rational number $a_{j} \neq 0$ such that

$$
\Gamma_{j}=\frac{\partial}{\partial \tau_{j}^{*}} .
$$


Corollary. If $u=u_{d}\left(\tau_{1}, \tau_{2}, \ldots, \tau_{d}\right)$ is expressed in terms of $\tau^{*}$ as $u=u_{d}^{*}\left(\tau_{1}^{*}, \ldots, \tau_{d}^{*}\right)$ then the function

$$
u=u_{d}^{*}\left(\tau_{1}^{*}+x, \tau_{2}^{*}, \ldots, \tau_{d}^{*}\right)
$$

is a solution of $\partial u / \partial \tau_{k}^{*}=X_{k} u$.

Proof. We proceed by induction as follows: Changing the notation of the $\tau_{j}$ also we assume that for some $k \geqq 1$ we have ${ }^{4}$

$$
\begin{aligned}
& \Gamma_{j}=\partial_{\tau_{j}}+\gamma_{j k} \partial_{\tau_{k}}, \quad 1 \leqq j \leqq k-1, \\
& \Gamma_{k}=\gamma_{k k} \partial_{\tau_{k}}
\end{aligned}
$$

up to terms $\partial_{\tau_{m}}$ for $m>k$, which will be suppressed. For $k=1$ this is trivially the case and for $k>1$ we will construct a transformation

$$
\left\{\begin{array}{l}
\tau_{j}^{*}=\tau_{j} \quad(j \neq k) \\
\tau_{k}^{*}=a_{k} \tau_{k}+g_{k}\left(\tau_{2}, \ldots, \tau_{k-1}\right)
\end{array}\right.
$$

such that

$$
\begin{aligned}
& \Gamma_{j}=\partial_{\tau_{j}^{*}}+0 \cdot \partial_{\tau_{k}^{*}} \\
& \Gamma_{k}=\partial_{\tau_{k}^{*}}
\end{aligned}
$$

up to terms $\partial_{\tau_{m}^{*}}$ with $m>k$. This is obviously sufficient for the proof, if we also verify that the $g_{k}$ and $a_{k}$ have the required properties.

To carry out this induction step it is convenient to break up (4.5) into $k$ steps and effectively make a second induction. First we achieve by $\tau_{k}^{*}=a_{k} \tau_{k}$ that $\Gamma_{k}$ $=\gamma_{k k} a_{k} \partial_{\tau_{k}^{*}}=\partial_{\tau_{k}^{*}}$ by setting $a_{k}=\gamma_{k k}^{-1}$, a rational number. Assume now that we already achieved

$$
\gamma_{j k}=0 \text { for } s<j<k \text {. }
$$

Then for $s<j<k$, using the commuting of the $X_{k}$, hence $\Gamma_{k}$, we compute

$$
0=\Gamma_{j} \Gamma_{s}-\Gamma_{s} \Gamma_{j}=\frac{\partial \gamma_{s k}}{\partial \tau_{j}} \partial_{\tau_{k}}
$$

hence $\gamma_{s k}$ depends on $\tau_{2}, \tau_{3}, \ldots, \tau_{s}$ only. Therefore we construct a transformation

$$
\tau_{k}^{*}=\tau_{k}+g\left(\tau_{2}, \ldots, \tau_{s}\right)
$$

so that

$$
\Gamma_{j}=\partial_{\tau_{j}} \text { for } \quad s<j \leqq k
$$

and

$$
\Gamma_{s}=\partial_{\tau_{s}^{*}}+\left(\frac{\partial g}{\partial \tau_{s}}+\gamma_{s k}\right) \partial_{\tau_{k}^{*}},
$$

$4 \quad$ We abbreviate $\partial / \partial \tau$ by $\partial_{\tau}$ 
while $\Gamma_{m}, 1 \leqq m \leqq s-1$, maintains its inductively assumed form. Thus, if we determine the polynomial $g$ such that

$$
\frac{\partial g}{\partial \tau_{s}}+\gamma_{s k}=0
$$

then we have effectively replaced $\gamma_{s k}$ by 0 . The choice of $g$ is unique if we require it to be isobaric. After finitely many steps we achieve

$$
\Gamma_{j}=\partial_{\tau_{j}^{*}}, \quad 1 \leqq j \leqq k,
$$

up to terms $\partial_{\tau_{m}}$ with $m>k$. This completes the induction argument. The isobaric character of the $g_{j}$ follows readily from that of the $\gamma_{k j}$. This completes the proof of Theorem 3.

As mentioned in the introduction the family of rational functions $u_{d}=$ $-2\left(\log \theta_{d}\right)^{\prime \prime}$ defines an embedding of the manifold $M_{d}$ of $[1]$ and the $\tau_{1}, \tau_{2}, \ldots, \tau_{d}$ can be viewed as parameters on this manifold. Therefore the roots $x_{1}, x_{2}, \ldots, x_{n}$, $n=n_{d}$ of $\theta_{d}$ :

$$
\theta_{d}=\prod_{j=1}^{n}\left(x-x_{j}\right)
$$

i.e. the poles of

$$
u_{d}=2 \sum_{j=1}^{n}\left(x-x_{j}\right)^{-2}
$$

satisfy, by the derivations in [1], the algebraic Equations (1.4). This fact could also be verified directly.

\section{A Nonselfadjoint Eigenvalue Problem}

The potential

$$
u_{d}=-2\left(\log \theta_{d}\right)^{\prime \prime}
$$

decay like $c x^{-2}$ at $\infty$ but may have a real singularity, at a zero of $\theta_{d}\left(x, \tau_{1}, \ldots, \tau_{d}\right)$. We will show

Lemma 6. For real values of $\tau_{1}, \ldots, \tau_{d}$ the polynomials $\theta_{d}, d \geqq 1$ possess a real root.

We postpone the proof. We can avoid such singularities by admitting complex values of $\tau_{1}, \tau_{2}, \ldots, \tau_{d}$ and choosing them so that no zero of $\theta_{d}(x, \tau)$ lies on the real $x$-axis, which is obviously possible. With such a complex potential we consider the operator

$$
L_{d}=-D^{2}+u_{d}
$$

in a dense domain in $L^{2}(-\infty,+\infty)$. Clearly this operator which depends on the parameters $\tau_{1}, \ldots, \tau_{d}$ is not selfadjoint and this gives rise to some anomalies to be described below. For studies of the inverse problem for such nonselfadjoint differential operators we refer to the work of Marčenko [13]. The main properties we wish to point out are that the potentials $u_{d}$ are all reflectionless and have a 
multiple eigenvalue at $\lambda=0$ for $\left(L_{d}\right)^{d}$. This is reflected in a higher order pole of the resolvent at $\lambda=0$.

We begin with describing the solutions of the eigenvalue equation

$$
L_{d} \phi=-s^{2} \phi
$$

thus $k=i^{-1} s$ would be the usual wave number. Using Lemma 4 and (2.19) we see that

$$
\psi(x, s)=T_{d} e^{s x}=\frac{W_{d}\left(e^{s x}\right)}{W_{d}}
$$

together with $\psi(x,-s)$ are solutions of (5.2). Here $e^{-s x} \psi(x, s)$ is a rational function of $s$ and $x$, in fact a polynomial of degree $d$ in $s$ as is clear from the last expression. Its asymptotic behavior for $x \rightarrow \infty$ is the same as for $\tau_{1}=\ldots=\tau_{d}=0$ as follows from the representation

$$
\begin{aligned}
& \quad \psi(x, s)=A_{d-1} A_{d-2} \ldots A_{0} e^{s x} \\
& \text { and } A_{j}=D-v_{j}=D+O(1 / x) \text {. Hence } \\
& \qquad e^{-s x} \psi(x, s)=s^{d}+O\left(x^{-1}\right) \text { for }|x| \rightarrow \infty ; s \neq 0 .
\end{aligned}
$$

Hence, for $s \neq 0$ the functions $\psi_{+}=\psi(x, s), \psi_{-}=\psi(x,-s)$ are linearly independent solutions of (5.2) and their Wronskian is the same as that of $s^{d} e^{s x},(-s)^{d} e^{-s x}$ :

$$
\left[\psi_{+}, \psi_{-}\right]=(-1)^{d} 2 s^{2 d+1} \text {. }
$$

For $\tau_{1}=\ldots=\tau_{d}=0$ the potential reduces to $u_{d}=d(d+1) x^{-2}$ and the functions $\psi(x, s)$ are proportional to the Bessel function

$$
\psi(x, i k)=a \sqrt{\frac{\pi}{2} k x H_{d+1 / 2}(k x) ; \quad a=(-1)^{d} i k^{d} .}
$$

It is well known that the Bessel functions for half integer subscripts are elementary functions. The potentials $u_{d}$ share this property with $d(d+1) x^{-2}$.

The Jost function is given by

$$
f(x, k)=(i k)^{-d} \psi(x, i k) \sim e^{i k x} \text { for } \quad x \rightarrow \infty .
$$

This function is a rational multiple of $e^{i k x}$, hence the reflection coefficient vanishes. Moreover, $\psi(x, k)$ is an entire function of $k$, but $f(x, k)$ has a pole of order $d$ at the origin since, by (2.22), (2.24)

$$
\psi(x, 0)=\frac{W_{d}(1)}{W_{d}}=(-1)^{d} \frac{W_{d-1}}{W_{d}}=\frac{\mu_{d}}{\mu_{d-1}}(-1)^{d} \frac{\theta_{d-1}}{\theta_{d}} \neq 0 .
$$

To get the solutions for $s=0$ we consider the Taylor expansion

$$
\psi(x, s)=\sum_{j=0}^{\infty} s^{j} \Phi_{j}(x)=T_{d} e^{s x}
$$

with

$$
\Phi_{j}=T_{d}\left(\frac{x^{j}}{j !}\right)
$$


Since $\left(L+s^{2}\right) \psi=0$ for $L=L_{d}$ we conclude that

$$
L \Phi_{j+2}+\Phi_{j}=0, \quad j \geqq 0 \quad \text { and } \quad L \Phi_{0}=L \Phi_{1}=0 .
$$

It turns out that the functions $\Phi_{2 i}$ are linearly independent since they have the asymptotic behavior

$$
\Phi_{2 i} \sim c_{i, d} x^{2 i-d} \text { for } x \rightarrow \infty
$$

and $c_{i, d} \neq 0$. However, $\Phi_{1}, \Phi_{3}, \ldots, \Phi_{2 d-1}$ are linearly dependent on $\Phi_{0}, \Phi_{2}, \ldots, \Phi_{2 d-2}$. In fact, we have the identities

$$
\Phi_{2 k-1}+\sum_{i=0}^{k-2} \varrho_{k-i} \Phi_{2 i}=0 \text { for } 2 \leqq k \leqq d
$$

and $\Phi_{1}=0$. Here $\varrho_{2}, \varrho_{3}, \ldots, \varrho_{d}$ are the parameters entering the definition $W_{d}$ through (2.25). Indeed, since

$$
T_{d} \chi=\frac{W_{d}(\chi)}{W_{d}}=W_{d}^{-1} W\left(\psi_{1}, \psi_{2}, \ldots, \psi_{d}, \chi\right)
$$

vanishes for $\chi=\psi_{k}$ we conclude from (2.25) and (5.5) immediately the validity of (5.8). Thus-by (5.6)-the equation

$$
L^{d} \phi=0
$$

has as solutions $\phi=\Phi_{0}, \Phi_{2}, \ldots, \Phi_{2 d-2}$ and, by (5.7),

$$
\Phi_{2 i} \in L^{2}(-\infty,+\infty) \text { for } 0 \leqq 2 i \leqq d-1 \text {. }
$$

This remark can be used to prove Lemma 6: Assume that $\theta_{d}$ has no real root. If $d \geqq 3$ then the null space of $L^{d}$ in $L^{2}(-\infty,+\infty)$ is at least 2 dimensional while that of $L$ is at most one-dimensional, since we are in the limit-point case. Therefore, for real $\tau$, when the operator $L$ has a selfadjoint extension, this is impossible if $u_{d}$ has no singularity. Hence $\theta_{d}$ must have a real zero for $d \geqq 3$. For $d=1,2$ this is evident which proves Lemma 6.

Thus the existence of generalized eigenfunctions of $L$ for $\lambda=0$ given by (5.6) are a reflection of the nonselfadjoint character of $L$ since $u_{d}$ is complex.

For completeness we describe a basis for the solutions of $L^{d} \phi=0$, since $\Phi_{0}, \Phi_{2}, \ldots, \Phi_{2 d-2}$ provide only half of such a basis. For this purpose we use the formula (2.25) to also define $\psi_{j}$ for $j>d$ setting $\varrho_{j}=0$ for $j>d$. Then we have

$$
L_{0}^{d} \psi_{k+d}=\left(-D^{2}\right)^{d} \psi_{k+d}=(-1)^{d} \psi_{k}
$$

and hence by (2.19) with $T=T_{d}$

$$
L^{d} T \psi_{k+d}=T L_{0}^{d} \psi_{k+d}=(-1)^{d} T \psi_{k}=0
$$

for $k=1,2, \ldots, d$. Hence $\phi=T \psi_{k+d}, k=1, \ldots, d$ are solutions of $L^{d} \phi=0$. This proves

Lemma 7. The null space of $L^{d}$ is spanned by

$$
\Phi_{2 i}=T\left(\frac{x^{2 i}}{(2 i) !}\right) \sim c_{i, d} x^{2 i-d} \quad(i=0, \ldots, d-1)
$$


and

$$
\psi_{k}=T\left(\psi_{k+d}\right) \sim b_{k, d} x^{2 k-1+d} \quad(k=1, \ldots, d) .
$$

One can, of course, not expect the usual orthogonality relations as in the selfadjoint case. For example one has for all $d \geqq 1$ for the eigenfunction $\Phi_{0} \in L^{2}$ the identity

$$
\int_{-\infty}^{+\infty} \Phi_{0}^{2} d x=0
$$

More generally, we show

Lemma 8. For $i+j<d, i, j \geqq 0$ one has

$$
\int_{-\infty}^{+\infty} \Phi_{2 i} \Phi_{2 j} d x=0
$$

and for $2 j<d-1$

$$
\int_{-\infty}^{+\infty} \Phi_{2 j} \psi(x, i k) d x=0 ; \quad(k \text { real })
$$

Proof. By (5.5) one finds by integration by parts

$$
\begin{aligned}
\Phi_{2 i} \Phi_{2 j} & =T\left(\frac{x^{2 i}}{(2 i) !}\right) T\left(\frac{x^{2 j}}{(2 j) !}\right) \\
& =\frac{x^{2 i}}{(2 i) !} \cdot\left(T^{*} T\right)\left(\frac{x^{2 j}}{(2 j) !}\right)+\frac{d}{d x} R,
\end{aligned}
$$

where $R$ is a rational function of $x$. Since $T^{*} T=L_{0}^{d}=\left(-D^{2}\right)^{d}$ and $j<d$ the first term on the right-hand side vanishes. From (5.7) we conclude that $R(x)$ $=O\left(x^{2 i+2 j-2 d+1}\right) \rightarrow 0$ for $|x| \rightarrow \infty$, hence

$$
\int_{-\infty}^{+\infty} \Phi_{2 i} \Phi_{2 j} d x=\left.R(x)\right|_{-\infty} ^{+\infty}=0 .
$$

The second relation uses the same argument.

We discuss briefly the spectral representation and the Green's function which can all be explicitly given. We simply present the relevant formulae:

If $\operatorname{Im} k>0$ and $\lambda=k^{2}$ then the equation

$$
\left(L_{d}-\lambda\right) \phi=h \in L^{2}(-\infty,+\infty)
$$

has the solution

$$
\phi(x)=\int_{-\infty}^{+\infty} G(x, y, k) h(y) d y,
$$

where the Green's function is given by

$$
G(x, y, k)=\left\{\begin{array}{lll}
\frac{\psi_{+}(x) \psi_{-}(y)}{-2 i k^{2 d+1}} & \text { for } & x>y \\
\frac{\psi_{-}(x) \psi_{+}(y)}{-2 i k^{2 d+1}} & \text { for } & x<y .
\end{array}\right.
$$


Here we write $\psi_{+}(x), \psi_{-}(x)$ as abbreviations for $\psi(x, i k), \psi(x,-i k)$ and use that $\psi_{+} \rightarrow 0$ for $x \rightarrow+\infty$, and $\psi_{-} \rightarrow 0$ for $x \rightarrow-\infty$. Thus $k^{2 d+1} G(x, y, k)$ is a polynomial in $k$, and at $k=0$ the Green's function has a pole of order $2 d+1$ in $k$.

We turn to the spectral representation of a complex valued function $h(x)$.

Theorem 4. If $\int_{-\infty}^{+\infty}\left(|x|^{d}+1\right)|h(x)| d x<\infty$ and $h \in C^{\prime}(-\infty,+\infty)$ then

$$
a_{+}(k)=\int_{-\infty}^{+\infty} h(x) \psi_{+}(x) d x ; \quad a_{-}(k)=\int_{-\infty}^{+\infty} h(x) \psi_{-}(x) d k
$$

for $k \geqq 0$ implies

$$
h(x)=\frac{1}{2 \pi} \int_{0}^{\infty}\left\{k^{-2 d}\left(a_{+}(k) \psi_{-}(x)+a_{-}(k) \psi_{+}(x)\right)\right\}_{\mathrm{reg}} d k .
$$

Here \{\}$_{\text {reg }}$ denotes the regular part at $k=0$ of the function in the parentheses.

Remark. It is necessary to take the regular part of the integrand to ensure convergence of the integrand. One could also interpret this integral as the "part fini" in the sense of Hadamard or as regularization of a singular integral as is standard in the theory of distributions [14]. In our case it means that one subtracts from $a_{+} \psi_{-}+a_{-} \psi_{+}$the first $2 d$ terms of the Taylor expansion

$$
\sum_{\nu=0}^{2 d-1} p_{v} k^{\nu}=\sum_{\alpha=0}^{d-1} p_{2 \alpha} k^{2 \alpha}
$$

since our function is even. In the present case it is easy to calculate $p_{2 \alpha}$ using the Taylor expansion of $\psi(x, s)=\sum s^{j} \Phi_{j}$. One finds

$$
\begin{gathered}
p_{2 \alpha}=\int_{-\infty}^{+\infty} P_{2 \alpha}(x, y) h(y) d y \\
P_{2 \alpha}(x, y)=2(-1)^{\alpha} \sum_{j=0}^{2 \alpha}(-1)^{j} \Phi_{2 \alpha-j}(x) \Phi_{j}(y) .
\end{gathered}
$$

In the case that $a_{+}(k), a_{-}(k)$ vanishes of order $2 d$ at the origin the above result follows in part from the Fourier transform. For this formal argument we assume $h \in C_{\text {comp }}^{d+1}$ so that $T^{*} h \in C_{\text {comp }}^{\prime}$. Applying the Fourier theorem to $T^{*} h$ we have: If

$$
a(k)=\int_{-\infty}^{+\infty}\left(T^{*} h\right) e^{i k x} d x=\int_{-\infty}^{+\infty} h \psi(x, i k) d x
$$

then the Fourier theorem gives

$$
T^{*} h=\frac{1}{2 \pi i} \int_{-\infty}^{+\infty} a(k) e^{-i k x} d k=\frac{1}{2 \pi i} \int_{0}^{\infty}\left(a_{+} e^{-i k x}+a_{-} e^{i k x}\right) d k,
$$

where $a_{+}(k)=a(k), a_{-}(k)=a(-k)$ for $k \geqq 0$. Using the identity $L_{0}^{d}=T^{*} T$ we find

$$
k^{2 d} e^{i k x}=L_{0}^{d} e^{i k x}=T^{*} \psi(x, i k)
$$

and the above identity takes the form

$$
T^{*}\left(h-\frac{1}{2 \pi i} \int_{0}^{\infty} k^{-2 d}\left(a_{+} \psi_{-}+a_{-} \psi_{+}\right) d k\right)=0
$$


provided that $a_{+}, a_{-}$vanishes of order $2 d$ at the origin. Next we observe that the null space of $T^{*}$ is spanned by $\Phi_{2 j}(j=0,1, \ldots, d-1)$. Indeed this null space has dimension $d$ as $T^{*}$ is a differential operator of order $d$ and since $L_{0}^{d}=T^{*} T$ we have

$$
T^{*} \Phi_{2 j}=T^{*} T\left(\frac{x^{2 j}}{(2 j) !}\right)=L_{0}^{d}\left(\frac{x^{2 j}}{(2 j) !}\right)=0 .
$$

Therefore we can write the last relation as

$$
h-\frac{1}{2 \pi i} \int_{0}^{\infty}\left(k^{-2 d}\left(a_{+} \psi_{-}+a_{-} \psi_{+}\right)\right)_{\mathrm{reg}} d k=\sum_{i=0}^{d-1} c_{i} \Phi_{2 i} .
$$

It remains to show that the $c_{i}$ vanish.

For this purpose we proceed differently. We first assume that $h \in C_{\text {comp }}^{1}$.

We apply the Cauchy integral formula to the function

$$
w(x, k)=\int_{-\infty}^{+\infty} G(x, y, k) h(y) d y+Q\left(k^{-1}\right),
$$

where $Q$ is an odd polynomial in $k^{-1}$ satisfying $Q=O\left(|k|^{-3}\right)$ at $\infty$ and which will be chosen presently. Since $w(x, k)$ is analytic in $\operatorname{Im} k>0$ we have

$$
\int_{C_{\delta}} w(x, k) k d k=0
$$

for any closed curve $C_{\delta}$ in the upper half plane. We choose $C_{\delta}$ as a semicircle centered at $i \delta ; \delta>0$ together with the diameter. One verifies from the asymptotic properties of $\psi_{+}, \psi_{-}$and using integration by parts that

$$
w(x, k) \sim-\frac{h(x)}{k^{2}} \text { as }|k| \rightarrow \infty,
$$

and this holds independently of the choice of $Q$. Therefore the integral taken over the upper semicircle tends to

$$
-h(x) \int \frac{k d k}{k^{2}}=-\pi i h(x)
$$

as the radius tends to infinity. Therefore we have

$$
\pi i h(x)=\int_{-\infty+i \delta}^{+\infty+i \delta} w(x, k) k d k=\int_{0+i \delta}^{\infty+i \delta}(w(x, k)-w(x,-k)) k d k .
$$

Next we let $\delta$ tend to zero. It is here that we have to take account of the singularity of $G(x, y, k)$ at $k=0$. The contribution to the integrand of $(5.11)$ is

$$
\begin{aligned}
& k \int_{-\infty}^{+\infty}(G(x, y, k)-G(x, y,-k)) h d y \\
& =-\frac{1}{2 i k^{2 d}}\left(\psi_{+} \int_{-\infty}^{x} \psi_{-} h d y+\psi_{-} \int_{x}^{\infty} \psi_{+} h d y+\psi_{-} \int_{-\infty}^{x} \psi_{+} h d y+\psi_{+} \int_{x}^{\infty} \psi_{-} h d y\right) \\
& =-\frac{1}{2 i k^{2 d}}\left[\psi_{+} \int_{-\infty}^{+\infty} \psi_{-} h d y+\psi_{-} \int_{-\infty}^{+\infty} \psi_{+} h d y\right] .
\end{aligned}
$$


In order to avoid the singularity at the origin we take the regular part of this expression. For this purpose we denote by $p(k)=\sum_{\alpha=0}^{d-1} p_{2 \alpha} k^{2 \alpha}$ the first terms in the Taylor expansion of the parentheses [ ] so that the regular part of the above expression is equal to

$$
k \int_{-\infty}^{+\infty}(G(x, y, k)-G(x, y,-k)) h(y) d y+\frac{1}{2 i} k^{-2 d} p(k)
$$

Thus if we set

$$
Q\left(k^{-1}\right)=\frac{1}{4 i} k^{-2 d-1} p(k)
$$

this is indeed an odd polynomial in $k^{-1}$ satisfying $Q=O\left(|k|^{-3}\right)$ and the last expression agrees with $k(w(x, k)-w(x,-k))$. This expression is regular at $k=0$ with this choice of $Q$ and for $\delta \rightarrow 0$ Equation (5.11) becomes

$$
\pi i h(x)=\frac{1}{-2 i} \int_{0}^{\infty}\left\{\frac{1}{k^{2 d}}\left[\psi_{+} \int_{-\infty}^{+\infty} \psi_{-} h d y+\psi_{-} \int_{-\infty}^{+\infty} \psi_{+} h d y\right]\right\}_{\mathrm{reg}} d k
$$

which proves (5.9) under the assumption $h \in C_{\text {comp }}^{\prime}$.

For the proof of the full statement one needs standard estimates for the integrand in (5.9) which we forego. But we wish to point out that for

$$
h=\Phi_{2 j}, \quad j<\frac{d-1}{2}
$$

we have by Lemma $8 a_{+}(k) \equiv 0 \equiv a_{-}(k)$. Thus these $h$ can clearly not be represented in the form (5.9). This is due to the fact that $\Phi_{2 j}$ decays at best like $x^{-d}$ and therefore violates the assumption of Theorem 4.

Corollary to Theorem 4. If $h, \tilde{h}$ are two functions satisfying the assumption of Theorem 4 and if $\tilde{a}_{ \pm}(k)$ are analogously defined as $a_{ \pm}(k)$ then one has the "Parseval equation"

$$
\int_{-\infty}^{+\infty} h(x) \tilde{h}(x) d x=\frac{1}{2 \pi} \int_{0}^{\infty}\left\{k^{-2 d}\left(a_{+} \tilde{a}_{-}+a_{-} \tilde{a}_{+}\right)\right\}_{\mathrm{reg}} d k .
$$

This follows easily by exchanging order of integration from Theorem 4.

The above relations (5.9), (5.12) are simple generalizations of the Fourier transform and the Parseval relation into which they go over for $d=0$. To get the analogue of the real form of the Fourier transform we set

$$
c(x, k)=T \cos k x ; \quad s(x, k)=T \sin k x
$$

so that

$$
\psi_{+}=c+i s, \quad \psi_{-}=c-i s .
$$


Accordingly we set

$$
\alpha(k)=\int_{-\infty}^{+\infty} h(x) c(x, k) d x, \quad \beta(k)=\int_{-\infty}^{+\infty} h(x) s(x, k) d x
$$

and obtain from (5.9)

$$
h(x)=\frac{1}{\pi} \int_{0}^{\infty}\left\{k^{-2 d}(\alpha c+\beta s)\right\}_{\mathrm{reg}} d k
$$

and for two functions $h, \tilde{h}$

$$
\int_{-\infty}^{+\infty} h \tilde{h} d x=\frac{1}{\pi} \int_{0}^{\infty}\left\{k^{-2 d}(\alpha \tilde{\alpha}+\beta \tilde{\beta})\right\}_{\mathrm{reg}} d k
$$

Thus with respect to this basis the spectral density matrix is

$$
d \varrho=\frac{1}{\pi k^{2 d}}\left(\begin{array}{cc}
d k & 0 \\
0 & d k
\end{array}\right)
$$

which is to be taken in the regularized sense [14].

\section{References}

1. Airault,H., McKean,H.P., Moser,J.: Rational and elliptic solutions of the Korteweg-deVries equation and a related many body problem. Comm. Pure Appl. Math. 30, 95-148 (1977)

2. Crum,M.M.: Associated Sturm-Liouville systems. Quart. J. Math., Ser. 2, 6, $121-127$ (1955)

3. Gardner,C.S., Greene,J.M., Kruskal,M.D., Miura, R. M. : Korteweg-deVries equation and generalizations. VI. Methods for exact solution. Comm. Pure Appl. Math. 27, 97-133 (1974)

4. Its, A.R., Matveev,V.B.: Hill's operator with finitely many gaps. Funct. Anal. Appl. 9, 69-70 (1975)

5. Lax,P.D.: Integrals of nonlinear equations of evolution and solitary waves. Comm. Pure Appl. Math. 21, 467-490 (1968)

6. McKean,H.P., Moerbeke,P.van: The spectrum of Hill's equation. Inventiones Math. 30, 217-274 (1975)

7. Miura, R.M.: Korteweg-deVries equation and generalization. I. A remarkable explicit nonlinear transformation. J. Math. Phys. 9, 1202-1204 (1968)

8. Miyake,M., Shimizu,K., Mugibayashi, N.: Soliton solutions of the Korteweg-deVries equation. J. Phys. Soc. Japan 37, 868-873 (1974)

9. Moses,H.E.: A solution of the Korteweg-deVries equation in a half-space bounded by a wall. J. Math. Phys. 17, 73-75 (1976)

10. Olver,P.: Evolution equations possessing infinitely many symmetries. Preprint (1976)

11. Wadati,M., Sanuki,H., Konno,K.: Relationships among inverse method, Bäcklund transformation and an infinite number of conservation laws. Progr. Theoret. Phys. 53, 419 (1975)

12. Deift,P.: Application of a commutation formula. Duke Math. J. (to appear) (1978)

13. Marčenko, V.: Mat. Sbornik 52 (94), 739-788 (1960)

14. Gelfand,I. M., Shilow, G.E.: Generalized functions I. § 3. New York: Academic Press 1960

15. Lax,P.D.: Almost periodic solutions of the KdV equation. SIAM Rev. 18, 351-375 (1976)

16. Moses,H.E., Tuan,S.F.: Potentials with zero scattering phase. Nuovo Cimento, Ser. 10, 13, 197206 (1959)

17. Ablowitz,M.J., Satsuma,J.: Solitions and rational solutions of nonlinear evolution equations (to appear)

18. Calogero,F.: Motions of poles and zeros of special solutions of nonlinear and linear partial differential equations and related "solvable" many-body problems. Preprint 45, University di Roma (May 1977) 
19. Adler,M., Moser,J.: On a class of polynomials connected with the Korteweg-deVries equation. MRC Report No. 1751, University of Wisconsin (May 1977)

20. Choodnovsky,D.V., Choodnovsky,G.V.: Pole expansions of nonlinear partial differential equations. Nuovo Cimento 40 B, 339-353 (1977)

21. Burchenall,J.L., Chaundy, T.W.: Commutative ordinary differential operators. Proc. London Soc., Ser. 2, 21, $420-440$ (1923)

Communicated by J. Moser

Received February 1, 1978 